\title{
Evaluation of postgastrectomy symptoms after distal gastrectomy with Billroth-I reconstruction using the Postgastrectomy Syndrome Assessment Scale-45 (PGSAS-45)
}

\author{
Kazunari Misawa $\cdot$ Masanori Terashima $\cdot$ Yoshikazu Uenosono $\cdot$ \\ Shuichi Ota $\cdot$ Hiroaki Hata $\cdot$ Hiroshi Noro $\cdot$ Kentaro Yamaguchi $\cdot$ \\ Hiroshi Yajima $\cdot$ Toshikatsu Nitta $\cdot$ Koji Nakada
}

Received: 26 March 2014/ Accepted: 13 July 2014/Published online: 5 August 2014

(c) The International Gastric Cancer Association and The Japanese Gastric Cancer Association 2014

\begin{abstract}
Background Distal gastrectomy with Billroth-I reconstruction (DGBI) is the most commonly used treatment approach for gastric cancer patients in Japan. The aim of this study was to assess and elucidate the effect of different surgical DGBI techniques on postgastrectomy syndrome (PGS) using the Postgastrectomy Syndrome Assessment Scale-45 (PGSAS-45).

Methods The newly created PGSAS-45 composed of 45 questions was used in this study. The scale was distributed to 2,922 patients who underwent gastrectomy $>1$ year prior. Completed forms were returned by 2,520 patients $(86 \%)$, of which 909 underwent DGBI. The effects of performing the Kocher maneuver, differences in the size of the gastric remnant and differences the anastomosis technique had on the main outcome measures of PGSAS-45 were analyzed.
\end{abstract}

For the Japan Postgastrectomy Syndrome Working Party.

\section{K. Misawa $(\bowtie)$}

Department of Gastroenterological Surgery, Aichi Cancer Center Hospital, 1-1 Kanokoden, Chikusa-ku, Nagoya 464-8681, Japan e-mail: misawakzn@aichi-cc.jp

M. Terashima

Division of Gastric Surgery, Shizuoka Cancer Center,

Nagaizumi-Cho, Japan

\section{Y. Uenosono}

Department of Digestive Surgery, Kagoshima University, Graduate School of Medicine, Kagoshima, Japan

\section{S. Ota}

Department of Surgery, Osaka Saiseikai Noe Hospital, Osaka, Japan

H. Hata

Department of Surgery, Kyoto Medical Center, Kyoto, Japan
Results Patients for whom the Kocher maneuver was performed experienced significantly worse meal-related distress and poorer quality of ingestion. Additionally, a less satisfactory physical and mental component summary from the SF-8 was reported. Patients with larger gastric remnants showed significantly better scores on the diarrhea subscale, a slightly better trend for the need for additional meals and dissatisfaction with eating. Regarding the anastomosis technique, there was no difference between the hand-sewn, circular stapler (CS) and linear stapler end-to-end anastomosis, and there was also no difference between the end-toend and side-to-end anastomosis with the CS.

Conclusions The Kocher maneuver may increase mealrelated distress, reduce the quality of ingestion and impart a negative effect on quality of life (QOL). The larger gastric remnants may cause reduction in diarrhea and an improvement in meal-related scores.

H. Noro

Department of Gastroenterological Surgery, Otemae Hospital, Osaka, Japan

K. Yamaguchi

Department of Surgery, Tokyo Women's Medical University

Medical Center East, Tokyo, Japan

H. Yajima

Department of Surgery, The Jikei University Kashiwa Hospital,

Kashiwa, Japan

T. Nitta

Department of Gastroenterological and Beast Center Surgery,

Shiroyama Hospital, Habikino, Japan

K. Nakada

Department of Surgery, The Jikei University School of

Medicine, Tokyo, Japan 
Keywords Postgastrectomy syndromes - Questionnaires · Quality of life · Gastrectomy

\section{Introduction}

Gastric cancer is the most prevalent form of cancer in Japan and in recent years the percentage of patients with early-stage cancer has increased. Gastrectomy is commonly performed and in the cases of early-stage cancer the prognosis is extremely satisfactory, with a 5-year survival rate of $\geq 90$ percent [1]. However, the issue of postgastrectomy syndrome (PGS) experienced by gastrectomy patients who have undergone radical therapy is a serious long-term problem. In addition to decreased oral intake and weight loss due to a reduction in the size of the stomach or total loss of the stomach, PGS symptoms such as dumping syndrome, which includes both systemic and abdominal symptoms, have an effect on long-term postoperative quality of life (QOL). Elucidating the status of PGS and the correlation with gastrectomy is important in the selection of appropriate surgical techniques and methods for the prevention and appropriate treatment of PGS.

A working group was formed in 2006 to standardize the assessment methods of PGS. This working group created the Postgastrectomy Syndrome Assessment Scale-45 (PGSAS-45) for use in comprehensive assessment of postgastrectomy symptoms, living status and QOL [2]. A nationwide, multi-institutional, collaborative, cross-sectional postgastrectomy syndrome assessment study was conducted using this scale. The present study assessed the effect of surgical techniques used in distal gastrectomy with Billroth-I reconstruction (DGBI) on PGS; DGBI is the most commonly used treatment approach for gastric cancer patients in Japan.

\section{Patients and methods}

Fifty-two member facilities of the Japanese Postgastrectomy Syndrome Working Party (JPGSWP) participated in this study. Patient inclusion criteria were as follows: (1) gastric cancer patients with pathologically confirmed stage IA or IB disease; (2) patients between 20 and 75 years; (3) patients who underwent gastrectomy for the first time; (4) patients who were not treated with chemotherapy; (5) patients without any recurrence or distant metastasis; (6) patients with an interval of one year or longer after gastrectomy; (7) physical status 0 or 1; (8) fully capable of understanding and responding to the questionnaire; (9) without any other disease or previous surgery which may influence the results of questionnaire more than gastrectomy; (10) without any organ failure or mental disease; and, (11) written informed consent with date and signature of the said person. Patients with active dual malignancy and who underwent concomitant resection of other organs due to another disease were excluded; co-resection equivalent to cholecystectomy was the exception.

We used the newly created PGSAS-45 to assess PGS in this study [2]. This scale is composed of questions pertaining to 45 items. This includes 8 items from the existing SF-8 scale [3], 15 items from the Gastrointestinal Symptom Rating Scale (GSRS) [4] and 22 items judged to be clinically important and newly selected by surgeons in the JPGSWP. Specifically, there are 8 items pertaining to general postgastrectomy symptoms, 2 items pertaining to the type and number of dumping syndromes, 8 items pertaining to the amount of oral intake and quality of ingestion, 1 item pertaining to working (job) conditions and 3 items pertaining to the level of dissatisfaction with daily life. The scale was distributed to the patients participating in this study and they returned the completed forms to the data center by mail. Factor analysis was performed on the data regarding standard gastrectomy (total gastrectomy and distal gastrectomy), and 23 symptom items of the data were clustered into the following 7 subscales (SSs): esophageal reflux, abdominal pain, meal-related distress, indigestion, diarrhea, constipation and dumping. In addition, the total symptom score, quality of ingestion SS, quantity of each meal, necessity for additional meals, dissatisfaction with symptoms, meals and work, as well as dissatisfaction with daily life SS, the physical component summary (PCS), the mental component summary (MCS) from the SF-8 and percentage of change in body weight were analyzed as the main outcome measures. The SS scores represented the average scores for the component items and the total symptom score was calculated from the average of the $7 \mathrm{SS}$ scores. Background data, including patient attributes such as age at the time of administration of the scale, sex, height, and weight, as well as the degree of lymphadenectomy, approach used, detailed surgical method, postoperative period, and other background data were collected from the patients' medical records. This study was registered with the University Hospital Medical Information Network (UMIN) Clinical Trials Registry (No. 000002116). This study was approved by the local ethics committees at each institution.

The scale was distributed to 2,922 patients between July 2009 and December 2010. Completed forms were returned by 2,520 patients $(86 \%)$. Of the 2,520 patients from whom data was obtained 152 were excluded from the study because of the following reasons: 90 were aged $>75$; $<1$ year had passed since surgery for 29; 8 had combined resections; and 25 were in the "other" category. Thus, 2,368 patients were determined to be qualified, of which 393 underwent total gastrectomy, 909 underwent DGBI, 
475 underwent distal gastrectomy with Roux-en-Y reconstruction, 313 underwent pylorus-preserving gastrectomy, 193 underwent proximal gastrectomy and 85 underwent local resection. In this study 909 patients who underwent DGBI were selected for the analysis and effects of performing the Kocher maneuver, differences in the size of the gastric remnant and differences in the anastomosis technique on the main outcome measures were analyzed. The patients whose data of each procedure could not be corrected from the patients' medical records were excluded from each analysis, thus the total number of patients in each analysis resulted in less than 909.

Comparison of the main outcome measures was performed using the $t$ test, with significant differences set at $p<0.05$. Multi-group comparisons were conducted using analysis of variance (ANOVA) and Bonferroni-Dunn multiple comparisons were used in cases in which $p<0.1$. When multiple comparisons were performed among 3 groups statistical significance was set at $p<0.0167$. As a measure of the meaning of the magnitude of the differences, Cohen's $d$, which is defined as the difference between two means divided by a standard deviation for the data, were calculated as the effect sizes. To interpret effect sizes we followed the suggestion of Cohen [5] and regarded a Cohen's $d \geq 0.2$ and $<0.5$ as being small, $\geq 0.5$ and $<0.8$ as moderate and $\geq 0.8$ as large. Statistical analyses were performed by the biostatisticians mainly using StatView for Windows Ver. 5.0 (SAS Institute Inc.).

\section{Results}

Patient and surgical background

The average patient age at the time of completing the questionnaires was 61.6 years and the average postoperative observation period was 40.7 months. Approximately 45 percent of the patients underwent laparoscopic surgery, and in approximately 15 percent patients the celiac branch of the vagus nerve was preserved. Table 1 shows additional patient details.

\section{Kocher maneuver}

Comparison of the main outcome measures was conducted between patients in whom the Kocher maneuver was performed and those in whom it was not. In the Kocher maneuver patients significantly worse mealrelated distress, poorer quality of ingestion and less satisfactory PCS and MCS were reported. No significant differences were observed for any of the other assessment items (Table 2).
Table 1 DGBI Patient demographic information in DGBI

\begin{tabular}{|c|c|}
\hline Number of patients & 909 \\
\hline Postoperative period (months) & $40.7 \pm 30.7$ \\
\hline BMI (preoperative) & $22.7 \pm 3.0$ \\
\hline BMI (at the study) & $20.9 \pm 2.8$ \\
\hline Age & $61.6 \pm 9.1$ \\
\hline Gender (male/female) & $594 / 311$ \\
\hline Approach (laparoscopic/open) & $415 / 489$ \\
\hline \multicolumn{2}{|l|}{ Extent of lymph node dissection } \\
\hline D2 & 319 \\
\hline D1b & 444 \\
\hline D1a & 119 \\
\hline D1 & 8 \\
\hline D1> & 4 \\
\hline None & 0 \\
\hline Celiac branch of vagus (preserved/divided) & $133 / 754$ \\
\hline \multicolumn{2}{|l|}{ Combined resection } \\
\hline Cholecystectomy & 80 \\
\hline Splenectomy & 0 \\
\hline Others & 4 \\
\hline None & 743 \\
\hline \multicolumn{2}{|l|}{ Size of gastric remnant } \\
\hline More than half & 29 \\
\hline Around one-third & 799 \\
\hline Around one-fourth & 61 \\
\hline Less than one-fifth & 0 \\
\hline
\end{tabular}

$D G B I$ distal gastrectomy with Billroth-I reconstruction

Size of remnant stomach

To assess the effect of the size of the gastric remnant on the main outcome measures gastric remnant sizes were divided into the following 3 groups: $\geq 1 / 2$, approximately $1 / 3$ and $\leq 1 / 4$. The size of the gastric remnant had a significant effect on the diarrhea SS and patients with larger gastric remnants showed better scores on the diarrhea SS (Table 3).

Multiple comparisons were conducted on the diarrhea SS, necessity for additional meals and dissatisfaction with meals. Compared with the $\leq 1 / 4$ stomach remnant group, the $\geq 1 / 2$ stomach remnant group scored significantly better on the diarrhea SS $(p=0.0025)$. Though the significance levels between the $\geq 1 / 2$ group and the $1 / 3$ group ( $p=0.033$ ), and between the $1 / 3$ group and the $\leq 1 / 4$ group ( $p=0.035)$ were marginal in the Bonferroni-Dunn multiple comparisons, the Cohen's $d$ values revealed small but clinically meaningful effect sizes (0.46 and 0.27 , respectively). There was no significant difference between the $\geq 1 / 2$ and $1 / 3$ groups for the necessity for additional meals or between the $\leq 1 / 4$ and $\geq 1 / 2$ groups for dissatisfaction with meal; however, in both cases the $\geq 1 / 2$ group 
Table 2 Comparison of the main outcome measures between patients in whom the Kocher maneuver was performed after DGBI and those in whom the Kocher maneuver was not performed

Integrated subscales are italicized in the table

Outcome measures with *: higher score indicating better condition

Outcome measures without $*$ : higher score indicating worse condition

Interpretation of effect size in Cohen's $d: \geq 0.20$ as small, $\geq 0.50$ as medium, $\geq 0.80$ as large

\begin{tabular}{|c|c|c|c|c|c|c|}
\hline \multirow[t]{2}{*}{ Main outcome measures } & \multicolumn{2}{|c|}{$\begin{array}{l}\text { With Kocher } \\
\text { maneuver }(n=74)\end{array}$} & \multicolumn{2}{|c|}{$\begin{array}{l}\text { Without Kocher } \\
\text { maneuver }(n=771)\end{array}$} & \multirow[t]{2}{*}{$p$ value } & \multirow[t]{2}{*}{ Cohen's $d$} \\
\hline & Mean & SD & Mean & SD & & \\
\hline \multicolumn{7}{|l|}{ (Symptoms) } \\
\hline Esophageal reflux subscale & 1.8 & 1.0 & 1.7 & 0.8 & $\geq 0.1$ & \\
\hline Abdominal pain subscale & 1.8 & 0.7 & 1.7 & 0.7 & $\geq 0.1$ & \\
\hline Meal-related distress subscale & 2.3 & 1.0 & 2.0 & 0.9 & 0.0244 & 0.26 \\
\hline Indigestion subscale & 2.1 & 0.9 & 2.0 & 0.8 & $\geq 0.1$ & \\
\hline Diarrhea subscale & 2.3 & 1.1 & 2.1 & 1.1 & $\geq 0.1$ & \\
\hline Constipation subscale & 2.4 & 1.1 & 2.2 & 1.0 & $\geq 0.1$ & \\
\hline Dumping subscale & 2.1 & 1.2 & 2.0 & 1.0 & $\geq 0.1$ & \\
\hline Total symptom score & 2.1 & 0.8 & 2.0 & 0.7 & $\geq 0.1$ & \\
\hline \multicolumn{7}{|l|}{ (Living status) } \\
\hline Change in body weight* & $-8.8 \%$ & $7.9 \%$ & $-7.8 \%$ & $8.1 \%$ & $\geq 0.1$ & \\
\hline Ingested amount of food per meal* & 7.1 & 1.9 & 7.2 & 2.0 & $\geq 0.1$ & \\
\hline Necessity for additional meals & 1.9 & 0.9 & 1.9 & 0.8 & $\geq 0.1$ & \\
\hline Quality of ingestion subscale* & 3.4 & 1.1 & 3.8 & 0.9 & 0.0006 & 0.40 \\
\hline Ability for working & 1.9 & 1.0 & 1.7 & 0.9 & $\geq 0.1$ & \\
\hline \multicolumn{7}{|l|}{ (QOL) } \\
\hline Dissatisfaction with symptoms & 1.7 & 0.8 & 1.8 & 0.9 & $\geq 0.1$ & \\
\hline Dissatisfaction at the meal & 2.3 & 1.2 & 2.2 & 1.1 & $\geq 0.1$ & \\
\hline Dissatisfaction at working & 1.8 & 1.0 & 1.7 & 0.9 & $\geq 0.1$ & \\
\hline Dissatisfaction for daily life subscale & 1.9 & 0.8 & 1.9 & 0.8 & $\geq 0.1$ & \\
\hline Physical component summary* & 49.3 & 7.1 & 50.7 & 5.3 & 0.0388 & 0.22 \\
\hline Mental component summary* & 48.5 & 5.9 & 50.0 & 5.6 & 0.0275 & 0.27 \\
\hline
\end{tabular}

showed a slightly better trend. No significant differences were observed for any of the other assessment items.

\section{Anastomosis technique}

We assessed whether sutured (hand-sewn) anastomosis $(n=336)$, CS anastomosis $(n=183)$ and linear stapler (LS) anastomosis $(n=66)$ had an effect on the main outcome measures for patients in whom end-to-end B-I reconstruction was performed between the remnant stomach and the duodenum. The method of anastomosis had no significant effect on the main outcome measures.

We assessed whether performing end-to-end $(n=183)$ or side-to-end $(n=295)$ anastomosis between the gastric remnant and the duodenum had any effect on the main outcome measures for patients who underwent B-I reconstruction using the CS. There was no statistically significant difference on the main outcome measures between two above-mentioned procedures.

\section{Discussion}

In Japan more than half of the gastric cancer cases occur in the distal stomach; thus, distal gastrectomy is the most commonly performed procedure. Performing anastomosis in only 1 location is simple and it is the most physiologically appropriate approach because it allows food to pass through the duodenum [6]. Therefore, B-I reconstruction is the preferred post-distal gastrectomy reconstruction technique. The purpose of this study was to assess and elucidate the effect of different surgical techniques on PGS in cases in which DGBI, the most commonly performed gastric cancer surgical procedure in Japan, was performed.

Regardless of whether surgery was performed or anticancer drugs were administered, the effectiveness of treatment and post-treatment symptoms were evaluated using a patient-reported outcome (PRO) health-related quality of life (HRQOL) assessment. HRQOL was measured using a questionnaire. However, to determine if the resulting data are clinically useful, the method's validity, reliability and reproducibility must be verified. Thus, we used EORTC QLQ-C30 [7, 8], QLQ-STO22 [9, 10], SF-36 [11], GSRS [4] and other QOL questionnaires whose reliability and validity have been verified. However, because these questionnaires were not designed to assess PGS, they may not be able to fully assess the symptoms that are particular to postgastrectomy patients or the functional disabilities in daily life that are experienced by postgastrectomy patients. Thus, to perform a comprehensive 
Table 3 Comparison of the main outcome measures between patients with different gastric remnant sizes after DGBI

\begin{tabular}{|c|c|c|c|c|c|c|c|c|c|}
\hline \multirow[t]{3}{*}{ Main outcome measures } & \multicolumn{6}{|c|}{ Size of gastric remnant } & \multirow{3}{*}{$\begin{array}{l}p \text { value } \\
\text { (ANOVA) }\end{array}$} & \multirow{3}{*}{$\begin{array}{l}p \text { value } \\
(\mathrm{B} / \mathrm{D})\end{array}$} & \multirow{3}{*}{ Cohen's $d$} \\
\hline & \multicolumn{2}{|c|}{$\geq 1 / 2(n=29)$} & \multicolumn{2}{|c|}{$\begin{array}{l}\text { Approximately } 1 / 3 \\
(n=799)\end{array}$} & \multicolumn{2}{|c|}{$\leq 1 / 4(n=61)$} & & & \\
\hline & Mean & SD & Mean & $\mathrm{SD}$ & Mean & SD & & & \\
\hline \multicolumn{10}{|l|}{ (Symptoms) } \\
\hline Esophageal reflux subscale & 1.6 & 0.8 & 1.7 & 0.8 & 1.8 & 0.8 & $\geq 0.1$ & & \\
\hline Abdominal pain subscale & 1.6 & 0.7 & 1.7 & 0.7 & 1.8 & 0.7 & $\geq 0.1$ & & \\
\hline Meal-related distress subscale & 2.0 & 0.9 & 2.0 & 0.9 & 2.2 & 0.8 & $\geq 0.1$ & & \\
\hline \multirow[t]{2}{*}{ Indigestion subscale } & 2.0 & 1.1 & 2.0 & 0.8 & 2.1 & 0.9 & $\geq 0.1$ & & \\
\hline & & & & & & & & 0.0333 (a) & 0.46 \\
\hline \multirow[t]{2}{*}{ Diarrhea subscale } & 1.7 & 0.8 & 2.1 & 1.1 & 2.4 & 1.2 & 0.0088 & $0.0345(b)$ & 0.27 \\
\hline & & & & & & & & $0.0025^{*}(\mathrm{c})$ & 0.72 \\
\hline Constipation subscale & 2.2 & 1.0 & 2.2 & 1.0 & 2.1 & 0.9 & $\geq 0.1$ & & \\
\hline Dumping subscale & 1.8 & 1.0 & 2.0 & 1.0 & 2.0 & 0.9 & $\geq 0.1$ & & \\
\hline Total symptom score & 1.8 & 0.7 & 2.0 & 0.7 & 2.0 & 0.7 & $\geq 0.1$ & & \\
\hline \multicolumn{10}{|l|}{ (Living status) } \\
\hline Change in body weight* & $-5.9 \%$ & $10.5 \%$ & $-8.0 \%$ & $8.1 \%$ & $-9.0 \%$ & $6.9 \%$ & $\geq 0.1$ & & \\
\hline Ingested amount of food per meal* & 7.9 & 1.9 & 7.1 & 2.0 & 6.9 & 1.8 & $\geq 0.1$ & & \\
\hline Necessity for additional meals & 1.5 & 0.6 & 1.9 & 0.8 & 1.8 & 0.7 & 0.0791 & 0.0246 (a) & 0.47 \\
\hline Quality of ingestion subscale* & 3.9 & 0.9 & 3.8 & 0.9 & 3.6 & 3.6 & $\geq 0.1$ & & \\
\hline Ability for working & 1.7 & 0.9 & 1.8 & 0.9 & 1.7 & 0.8 & $\geq 0.1$ & & \\
\hline \multicolumn{10}{|l|}{ (QOL) } \\
\hline Symptoms dissatisfaction & 1.8 & 0.9 & 1.8 & 0.9 & 2.0 & 0.9 & $\geq 0.1$ & & \\
\hline Meal dissatisfaction & 1.8 & 1.0 & 2.2 & 1.1 & 2.3 & 1.0 & 0.0821 & $0.0371(\mathrm{c})$ & 0.52 \\
\hline Work dissatisfaction & 1.6 & 0.8 & 1.7 & 0.9 & 1.7 & 0.9 & $\geq 0.1$ & & \\
\hline Dissatisfaction for daily life subscale & 1.7 & 0.8 & 1.9 & 0.8 & 2.0 & 0.8 & $\geq 0.1$ & & \\
\hline Physical component summary* & 51.0 & 4.8 & 50.5 & 5.6 & 50.8 & 5.2 & $\geq 0.1$ & & \\
\hline Mental component summary* & 51.7 & 5.4 & 49.8 & 5.7 & 50.3 & 5.0 & $\geq 0.1$ & & \\
\hline
\end{tabular}

Integrated subscales are italicized in the table

Outcome measures with $*$ : higher score indicating better condition

Outcome measures without $*$ : higher score indicating worse condition

ANOVA: one-way analysis of variance; B/D: Bonferroni/Dunn multiple comparisons; (a) $\geq 1 / 2$ vs. approximately $1 / 3$; (b) approximately $1 / 3$ vs. $\leq 1 / 4 ;$ (c) $\geq 1 / 2$ vs. $\leq 1 / 4$

In ANOVA a $p$ value less than 0.05 was considered statistically significant

In Bonferroni/Dunn multiple comparisons a $p$ value $<0.0167$ was considered statistically significant

Interpretation of effect size in Cohen's $d$ : $\geq 0.20$ as small, $\geq 0.50$ as medium, $\geq 0.80$ as large

assessment of PGS, JPGSWP developed the PGSAS-45 and assessed its validity [2]. In addition, this scale was also used to compare PGS between Billroth-I and Roux-en-Y procedures following distal gastrectomy [12]. The present study used PGSAS-45 to assess the effect of the Kocher maneuver, the size of the gastric remnant and the anastomosis technique on postgastrectomy symptoms, living status and QOL in cases in which DGBI was performed.

The Kocher maneuver is a procedure in which an incision is made in the retroperitoneum on the right side of the descending duodenum, and then the duodenum and the head of the pancreas are mobilized to the left. It was originally reported as a maneuver to mobilize the duodenum that was performed by Kocher during a gastroduodenal anastomosis in 1903 [13, 14]. The Kocher maneuver is sometimes performed during gastrectomy for gastric cancer to confirm enlargement of and sample the paraaortic lymph node and to reduce the strain on the anastomotic site when performing B-I reconstruction. However, there have been no studies on the effect of the Kocher maneuver on PGS. The present study showed that performance of the Kocher maneuver results in poor scores for meal-related distress and quality of ingestion SS as well as poor scores for PCS and MCS from the SF-8. Meal-related 
distress is composed of the following symptom items: sense of food sticking, postprandial fullness and early satiation. As a result of the Kocher maneuver, the duodenum is moved to the left of its normal position, is elongated [13] and lies on the abdominal side when the patient is in a supine position. In other words, the duodenum locates higher than the gastric remnant. Such a morphological physical change may affect gastric emptying and other functions of the gastric remnant and increase meal-related distress. However, the specific mechanisms are currently unknown. In the future, elucidation of this mechanism through the gastrointestinal function tests such as a gastric emptying study would be informative. On the other hand, it is currently difficult to provide a proper explanation for why the Kocher maneuver leads to poor scores on the quality of ingestion SS that is composed of appetite, hunger, and satiety, as well as on PCS and MCS. It is possible that factors other than the explanatory variables assessed in this study may be involved. This issue will require further investigation. The Kocher maneuver is a useful technique when there is tension on the sutures of the anastomotic site of a B-I reconstruction; however, PGS could be worsened by employing this technique, therefore it should be applied minimally when it is required.

Regarding the extent of resection of the gastric wall, the Japanese gastric cancer treatment guidelines [15] stipulate "Standard gastrectomy involves resection of at least two-thirds of the stomach with D2 lymph node dissection". The guidelines also mention non-standard gastrectomy in which the extent of gastric resection and/ or lymphadenectomy is altered according to the tumor characteristics. In cases of early-stage gastric cancer modified surgery is performed, in which the extent of gastric resection and/or lymph node dissection is reduced. However, few studies have investigated the relationship between the size of the gastric remnant and PGS. Nomura et al. reported that in cases of early-stage gastric cancer, compared with the $1 / 2$ and $1 / 3$ gastric remnant DGBI patient group, patients in the $1 / 2$ gastric remnant group showed improved food intake, little postoperative weight loss and few abdominal symptoms such as diarrhea and abdominal pain [16]. It is believed that in cases of B-I reconstruction if the gastric remnant is large the gastric reservoir capacity is preserved, which helps to maintain a nutritional status and body composition. In the present study the larger the gastric remnant the lower was the percentage of weight loss. However, this result was not statistically significant. In the study by Nomura et al. weight measurements were taken $<1$ year after surgery; however, in the present study, weight measurements were taken on average $>3$ years after surgery. The present study includes data showing improvement in postoperative weight loss for a long postoperative period; therefore, we believe that there was no significant difference in the percentage of weight loss because of the size of the gastric remnant.

On the other hand, patients with larger gastric remnants showed improved scores on the necessity for additional meals and the meal dissatisfaction SS. Scores on the diarrhea SS were also significantly better. Our comparison of the $\geq 1 / 2$ gastric remnant group and the $\leq 1 / 4$ gastric remnant group showed a moderate effect on the diarrhea SS scores. There are multiple explanations regarding the causes of postgastrectomy diarrhea [17]. One of these is rapid gastric emptying. Because foods with high osmolality immediately flow directly into the small intestine after ingestion in postgastrectomy patients it is believed that intestinal peristalsis is accelerated and intestinal contents increase, resulting in diarrhea [18]. It is therefore highly possible that smaller gastric remnants and a reduced gastric reservoir capacity result in increased diarrhea. The influence of these factors is considered to result in persistent diarrhea for a relatively long postoperative period. The results of this study thus show that it is possible for patients with larger gastric remnants to have satisfactory PGS scores over a long term when DGBI is performed as long as there are no oncological problems such as early-stage gastric cancer.

Advances in automatic suturing devices and the widespread use of laparoscopic surgery have led to development and use of a variety of anastomosis methods even when BI reconstruction is performed after distal gastrectomy. However, there is no clear evidence indicating whether hand sewing or machine sewing is preferable or end-to-end or side-to-end anastomosis is preferable and debate over these issues continues. Few studies have investigated the influence of the method or type of anastomosis (side-toside vs. end-to-end) on PGS in cases of B-I reconstruction after distal gastrectomy. Kaiho et al. [19] compared sideto-end anastomosis with end-to-end anastomosis and reported that although side-to-end anastomosis showed satisfactory motility in the gastric remnant, there was no difference in gastric emptying. Takahashi et al. [20] compared mechanically stapled anastomosis (side-to-end) with hand-sewn anastomosis (end-to-end) and found that, although there was more residual stomach content in cases of mechanical stapled anastomosis when investigated using the Residue, Gastritis, Bile (RGB) classification via endoscopy 1 year after surgery, there was no significant difference in the frequency of gastritis and bile reflux. Endoscopic findings were not investigated in this study and the time from surgery until completion of the questionnaire was long, with an average of $>3$ years after surgery. A long time after surgery, the effect of the anastomotic technique on PGS, living status and QOL is either non-existent or minimal. 
This study has some limitations. Since the nature of this study was a retrospective explanatory study rather than a randomized controlled trial there was a certain imbalance in the number of patients and also a possible difference in patient characteristics among groups. Another limitation is that it was difficult to provide rational explanations for all results. PGS varies widely among individuals and is influenced by a variety of physical and functional factors. However, there have been no multi-institutional collaborative studies that have attempted to examine as many symptoms as this study for a single surgical procedure. Because the results of this study are generally consistent with our clinical experience we believe the results are reliable to a certain extent. In addition, the data from this study are the results of assessments of symptoms $\geq 1$ year after surgery and indicate PGS observed over a relatively long period of $\geq 3$ years after surgery. We believe that these data are extremely valuable in the quest to understand the long-term postoperative effects of techniques using DGBI. To determine the most appropriate gastrectomy technique from a PGS perspective investigation of the underlying pathophysiology via gastrointestinal function tests, such as a gastric emptying study, and further prospective studies, such as a randomized controlled trial, focusing on these issues may be required.

In conclusion, we performed an assessment of postDGBI long-term symptoms using the PGSAS-45. The results of this study indicate that in cases of DGBI the performance of the Kocher maneuver increases meal-related distress, reduces the quality of ingestion and imparts a negative effect on QOL. In addition, the data suggest that larger gastric remnants may reduce diarrhea and improve meal-related scores. On the other hand, we found no evidence that differences in the method or type of anastomosis had an effect on postgastrectomy disorders.

Acknowledgments This study was supported by a grant from the Jikei University and the Japanese Society for Gastro-surgical Pathophysiology. This study was conducted by JPGSWP. The authors thank all physicians who participated in this study and the patients whose cooperation made this study possible.

\section{References}

1. Nashimoto A, Akazawa K, Isobe Y, Miyashiro I, Katai H, Kodera Y, et al. Gastric cancer treated in 2002 in Japan: 2009 annual report of the JGCA nationwide registry. Gastric Cancer. 2013;16:1-27.

2. Nakada K, Ikeda M, Takahashi M, Kinami S, Yoshida M, Uenosono $\mathrm{Y}$, et al. Characteristics and clinical relevance of postgastrectomy syndrome assessment scale (PGSAS)-45: newly developed integrated questionnaires for assessment of living status and quality of life in postgastrectomy patients. Gastric Cancer. 2014;. doi:10.1007/s10120-014-0344-4.

3. Turner-Bowker DM, Bayliss MS, Ware JE Jr, Kosinski M. Usefulness of the SF-8 health survey for comparing the impact of migraine and other conditions. Qual Life Res. 2003;12:1003-12.

4. Svedlund J, Sjodin I, Dotevall G. GSRS-a clinical rating scale for gastrointestinal symptoms in patients with irritable bowel syndrome and peptic ulcer disease. Dig Dis Sci. 1988;33:129-34.

5. Cohen J. Statistical power analysis for the behavioral sciences. 2nd ed.: Lawrence Erlbaum Assoc.; 1988.

6. Kim BJ, O'Connell T. Gastroduodenostomy after gastric resection for cancer. Am Surg. 1999;65:905-7.

7. Kobayashi K, Takeda F, Teramukai S, Gotoh I, Sakai H, Yoneda $\mathrm{S}$, et al. A cross-validation of the European Organization for Research and Treatment of Cancer QLQ-C30 (EORTC QLQC30) for Japanese with lung cancer. Eur J Cancer. 1998;34:810-5.

8. Aaronson NK, Ahmedzai S, Bergman B, Bullinger M, Cull A, Duez NJ, et al. The European Organization for Research and Treatment of Cancer QLQ-C30: a quality-of-life instrument for use in international clinical trials in oncology. J Natl Cancer Inst. 1993;85:365-76.

9. Morita S, Kaptein AA, Oba K, Sakamoto J. The domain structure of the EORTC QLQ-STO22 supported by Japanese validation data. Psychooncology. 2008;17:474-9.

10. Blazeby JM, Conroy T, Bottomley A, Vickery C, Arraras J, Sezer $\mathrm{O}$, et al. Clinical and psychometric validation of a questionnaire module, the EORTC QLQ-STO 22, to assess quality of life in patients with gastric cancer. Eur J Cancer. 2004;40:2260-8.

11. Ware JE Jr. Sherbourne CD. The MOS 36-item short-form health survey (SF-36). I. conceptual framework and item selection. Med Care. 1992;30(6):473-83.

12. Terashima M, Tanabe K, Yoshida M, Kawahira H, Inada $T$, Okabe $\mathrm{H}$, et al. Postgastrectomy Syndrome Assessment Scale (PGSAS)-45 and changes in body weight are useful tools for evaluation of reconstruction methods following distal gastrectomy. Ann Surg Oncol. 2014. doi:10.1245/s10434-014-3583-z.

13. Elmslie RG. The Kocher manoeuvre. Aust N Z J Surg. 1973;42(4):345-8.

14. Kocher T. Mobilisierung des Duodenum und Gastroduodenostomie. Zbl Chir. 1903;2:33-40.

15. Japanese Gastric Cancer. A: Japanese gastric cancer treatment guidelines 2010 (ver. 3). Gastric Cancer. 2011;14(2):113-23.

16. Nomura E, Lee SW, Bouras G, Tokuhara T, Hayashi M, Hiramatsu M, et al. Functional outcomes according to the size of the gastric remnant and type of reconstruction following laparoscopic distal gastrectomy for gastric cancer. Gastric Cancer. 2011;14:279-84.

17. Cooperman AM. Postgastrectomy syndromes. Surg Annu. 1981;13:139-61.

18. Carvajal SH, Mulvihill SJ. Postgastrectomy syndromes: dumping and diarrhea. Gastroenterol Clin North Am. 1994;23:261-79.

19. Kaiho T, Gunji Y, Hirayama N, Hayashi H, Miyazaki S, Shimada $\mathrm{H}$, et al. Effect of the anastomosis-site after Billroth I gastrectomy on the motility of the remnant stomach. Hepatogastroenterology. 2004;51:906-11.

20. Takahashi T, Saikawa Y, Yoshida M, Otani Y, Kubota T, Kumai $\mathrm{K}$, et al. Mechanical-stapled versus hand-sutured anastomoses in billroth-I reconstruction with distal gastrectomy. Surg Today. 2007;37:122-6. 